\title{
La reinserción en la escuela y su vinculación con la vida familiar y laboral de tres generaciones en México
}

\author{
School reentry and its link to family and working life of three \\ generations in Mexico
}

\section{Eunice Danitza Vargas-Valle y Pedro Paulo Orraca-Romano}

\author{
Departamento de Estudios de Población, Departamento de Estudios \\ Económicos-El Colegio de la Frontera Norte, Tijuana, México
}

\section{Resumen}

A partir de la Encuesta Demográfica Retrospectiva (EDER) 2011, este trabajo analiza los niveles de reingreso a la escuela de tres generaciones en México y los factores condicionantes de éste, así como el nivel de estudios y el tipo de ocupación alcanzados con esta inversión. Se utilizan tablas de vida, modelos logísticos de tiempo discreto y estadística descriptiva. Este trabajo muestra la importancia de la baja intensidad del trabajo, del empleo en el sector público y de la etapa de crianza de los hijos para el retorno escolar, así como la posibilidad de obtener un mayor logro escolar y un mejor trabajo tras esta inversión en capital humano. Los resultados apoyan la importancia de políticas educativas encaminadas al regreso a la escuela de la juventud.

Palabras clave: Escolaridad, empleo, hijos, trayectorias, juventud.

Abstract

Based on the 2011 Retrospective Demographic Survey (EDER 2011, in Spanish), this paper analyzes the school reentry of three generations in Mexico and its associated factors, as well as the level of education attained and type of occupation after school reentry. To this end, life tables, discrete-time logistic models and descriptive statistics are employed. The results show the importance of a low-intensity job, employment in the public sector, and the child-rearing stage for school reentry, as well as the opportunity that this investment in human capital provides to achieve higher educational attainment and secure a better job. The results support the importance of education policies that promote school reentry for young people.

Key words: Schooling, employment, children, trajectories, youth. 


\section{INTRODUCCIÓN}

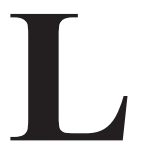

a educación formal tuvo una notable expansión en los últimos 60 años en México. Mientras que sólo la educación primaria se otorgó de manera obligatoria y gratuita en los primeros años, poco a poco la expansión alcanzó los niveles de educación secundaria y media superior. Así, las tres generaciones estudiadas en este artículo vivieron dicha expansión en distintos niveles. La primera generación, 1951-53, se favoreció de los efectos de las políticas educativas enfocadas a la universalización de la educación primaria (Mier y Terán y Rabell, 2001); la segunda generación, 1966-68, experimentó la expansión de la educación secundaria de los años 70, así como la desaceleración del crecimiento de la matrícula escolar tras las crisis económicas de los años 80 (SEP, 2007). Por último, la generación 1978-80 se benefició de la obligatoriedad de la secundaria de 1993 y la expansión de la media superior. ${ }^{1}$ Para 1995, año en el que esta generación había vivido las edades de escolarización obligatoria, en México ya se había alcanzado prácticamente la universalización de la educación primaria, 75 por ciento de cobertura en secundaria y 40 por ciento de cobertura en el nivel medio superior (SEP, 2013).

A la par que se expandió la oferta educativa, la demanda por la educación formal se amplió ante el cambio en el significado de la infancia y la disminución de la fecundidad, así como los requerimientos del empleo en las ciudades, en particular en la industria, la educación y los servicios (Gaxiola et al., 1997). Sin embargo, las transiciones a la adultez de diversos grupos sociales con menores capitales económicos, sociales y culturales estuvieron marcadas por la salida del sistema escolar y el inicio de la unión y la maternidad o el empleo remunerado a edades tempranas. El abandono escolar típico ocurrió a distintas edades en las tres generaciones estudiadas:

De los jóvenes de la cohorte más antigua (1951-1953), la mitad abandonaba la escuela una vez concluida la primaria. En las cohortes 1966-1968 y 19781980, el abandono escolar ocurría de forma más lenta y el riesgo aumentaba una vez concluida la secundaria (Rabell y Murillo, 2016: 307).

Ante el problema del abandono escolar, a lo largo de este medio siglo y especialmente con la promulgación de la Ley Nacional de Educación para

${ }^{1}$ La obligatoriedad de la media superior tuvo lugar hasta 2012, un año posterior al levantamiento de la encuesta utilizada. 
Adultos a partir de los años 70 , se intentaron crear planes escolarizados para mayores de 15 años en horarios accesibles, así como educación abierta para la acreditación de la educación básica. Estos programas educativos estaban orientados a aquellos individuos que habían desertado la escuela o no habían tenido la oportunidad de estudiar (Gaxiola et al., 1997). Además, en los años 90 se crearon telesecundarias para satisfacer la demanda de educación media en lugares remotos entre poblaciones marginadas, así como una serie de becas y programas compensatorios para mejorar la retención y promover la reincorporación a la escuela (SEP, 2004). Por ejemplo, tal ha sido el alcance de los programas de becas que para el año 2012 cerca de 46 por ciento de los estudiantes de bachilleratos públicos tenían una beca federal (SEP, 2012).

Con las acciones del Estado en un contexto educativo donde las desigualdades de origen y de género marcan fuertemente las trayectorias juveniles, la posibilidad de regresar a la escuela también se amplió. Así, Pérez y Lindstrom (2014) documentaron, con base en la Encuesta Demográfica Retrospectiva (EDER 2011) levantada en el año 1998, que las generaciones 1966-68 y 1951-53 regresaron con más frecuencia a la escuela que la generación 1936-38. Los autores destacaron también que regresar a la escuela estuvo vinculado a la inversión en capital humano para la obtención de una mejor posición socioeconómica y al curso de vida (al ser más frecuente entre los no unidos). No obstante, los autores no encontraron diferencias en cuanto al estatus laboral y la presencia de niños en el hogar, factores en los que nos enfocamos en este estudio.

Haciendo uso de la información biográfica de la EDER 2011 (EL COLEF, INEGI y UABC, 2013), este artículo analiza los niveles de regreso a la escuela de tres generaciones ${ }^{2}$ en México (1951-53, 1966-68 y 1978-80), así como los factores condicionantes de este fenómeno, haciendo énfasis en las características del empleo previo al retorno y la crianza de los hijos. En particular en este trabajo nos interesa explorar cómo las obligaciones familiares y las oportunidades de ciertos empleos se asocian a la trayectoria de retorno escolar en México. Además, se exploran los logros alcanzados en el tipo de ocupación, el sector de actividad y el nivel de estudios una vez que se concluye el periodo de retorno escolar. La EDER 2011, a comparación de la EDER 1998, permite ampliar el estudio de la asociación entre la duración de la jornada del trabajo que se desempeña y el regreso

\footnotetext{
${ }^{2}$ Población menor de 33 años, hasta estas edades la encuesta provee datos de las tres generaciones.
} 
a la escuela, además de que incluye información sobre la cohorte de nacimiento 1978-80.

Este estudio parte de una serie de hipótesis de trabajo vinculadas a la idea de la interdependencia de las esferas de actividad familiar, laboral y educativa en la vida de los jóvenes y la incidencia de los roles y las responsabilidades que se han adquirido a lo largo del curso de vida en los nuevos roles que se asumen (Bradburn, Moen y Dempster-McClain, 1995). La primera hipótesis es que los individuos pueden haber regresado a la escuela por las oportunidades de movilidad ocupacional de ciertos empleos o las campañas públicas a favor de la escolarización simultánea al trabajo, en una etapa histórica en México en que se expandió la obligatoriedad de la educación en distintos niveles. Por lo tanto, regresarían más a la escuela los empleados en el sector formal que en el informal, así como en los empleos no manuales, respecto a los manuales.

La segunda hipótesis está vinculada a la posibilidad de compatibilizar la escuela con el trabajo. Suponemos que los trabajos de tiempo completo, en comparación con los empleos de menor tiempo, repercuten negativamente en las posibilidades de retornar a la escuela, ya que se asume que la menor intensidad del trabajo y la posible mayor flexibilidad horaria que esto implica, puede significar una menor tensión entre el trabajo y la escuela.

Por último, la tercera hipótesis también está ligada a la dificultad para realizar roles simultáneos, en este caso, ser estudiante con criar a los hijos. Asumimos que para los desertores que tuvieron hijos era más sencillo regresar a la escuela cuando las demandas de cuidados - tanto en tiempo como en costos - eran menores, suponiendo un mayor reingreso a la escuela de los padres con la incorporación de los hijos al sistema escolar.

El artículo se estructura de la siguiente manera. En el primer apartado, se discuten los antecedentes teóricos y empíricos del problema de investigación. En el segundo apartado, se describe la metodología empleada para el análisis de la encuesta mencionada. En la tercera sección, se exponen los resultados que incluyen las tendencias y los perfiles de los jóvenes que regresan a la escuela, los factores asociados a este fenómeno y las consecuencias en los niveles de escolaridad y el tipo de empleo de haber regresado a la escuela. Finalmente, se discuten los hallazgos obtenidos.

\section{ANTECEDENTES TEÓRICOS Y EMPÍRICOS DE LOS CONDICIONANTES DEL REGRESO A LA ESCUELA}

De acuerdo a la perspectiva del curso de vida, en las sociedades modernas la transición a la adultez estuvo marcada por una serie de eventos que 
tendían a experimentarse en orden secuencial como son la salida de la escuela, el inicio de la vida laboral, la entrada en unión y el nacimiento del primer hijo; pudiendo la trayectoria ser diferenciada por sexo, ya que en algunos sectores sociales es aceptable que las mujeres pasen directamente de estudiantes a esposas y madres (Hogan, 1978; Elder, 1998; Hogan y Astone, 1986; Elder, Johnson y Crosnoe, 2003). No obstante, el curso de vida en tiempos más recientes ha tendido a ser más flexible y permitido trayectorias heterogéneas e incluso sincrónicas, algunas reversibles como el caso del regreso a la escuela (Settersten, 2003; Du Bois-Reymond y López Blasco, 2004; Hostetler, 2008).

Un aspecto importante de la perspectiva de curso de vida es que si bien reconoce al ser humano como constructor de su propia trayectoria de vida, también considera que las acciones humanas están constreñidas por el contexto social (Elder, Johnson y Crosnoe, 2003). Por lo tanto, la temporalidad y la secuencia de los eventos que marcan la transición a la adultez están reguladas por las instituciones sociales como la familia, la escuela y el mercado laboral. Son estas instituciones las que ofrecen las estructuras que brindan oportunidades y permiten el desarrollo de trayectorias comunes en cierto momento histórico. Además, a través de estas instituciones, la sociedad impone al individuo un conjunto de expectativas sobre los roles que debe realizar a determinada edad y sobre las edades en las que debe transitar hacia el desempeño de otros roles. De manera que los arreglos que hacen las instituciones sociales en apoyo a las transiciones a la adultez se hacen por lo regular para un determinado patrón normativo (Hogan y Astone, 1986; Harley y Mortimer, 2000; Elder, Johnson y Crosnoe, 2003).

En este sentido, Hogan (1978: 574) sostuvo que aun cuando las instituciones ofrecían oportunidades para experimentar trayectorias no lineales, estos arreglos eran solo remediales, ya que no eliminaban las desigualdades que se producen al estar fuera de los patrones normalizados de las trayectorias, pues existen "relojes sociales" que fijan la temporalidad y la secuencia de los eventos. Desigualdades que pueden estar condicionadas por las inequidades de origen social, las oportunidades educativas y de empleo y el grado de valoración de la educación formal en el mercado laboral. Sin embargo, otros autores como Settersten (2003) argumentaron que en tiempos de cambio social la evidencia era escasa y no concluyente sobre las ventajas de seguir una biografía estándar, ya que los individuos tienen que ajustarse a nuevas situaciones siguiendo otras opciones de formación familiar para navegar las dinámicas de las esferas de la educación y el trabajo. 
En realidad, los modelos lineales de transición a la vida adulta se han debilitado con el paso del tiempo debido a varios factores como: la extensión de la edad a la que se completa la educación formal, la falta de las garantías de empleo y protección social y la creciente responsabilidad de los jóvenes de construir sus propias biografías (Du Bois-Reymond y López Blasco, 2004). Por ello, los modelos normativos de curso de vida se han desestandarizado, ya que los jóvenes experimentan los eventos que marcan la adultez a edades más tardías, los itinerarios vitales son menos lineales y las trayectorias a la adultez son cada vez más individualizadas y menos el producto de modelos colectivos (Du Bois-Reymond y López Blasco, 2004). Desafortunadamente, por la incertidumbre del contexto, el individuo asume el riesgo de optar por determinada trayectoria dentro de la gama de opciones presentes en la sociedad, enfrentando barreras estructurales.

En este sentido, el regresar a la escuela, cuando se han asumido roles laborales o familiares, es una de estas trayectorias reversibles de transición a la adultez, cuya elección implica asumir un conjunto de riesgos. Las instituciones pueden ofrecer arreglos para la secuencia desordenada de los eventos que definen la transición a la adultez, por ejemplo, para estar simultáneamente participando en la escuela y el mercado laboral o para ejercer la maternidad o la paternidad mientras se estudia, como son los programas que permiten la conclusión del nivel escolar que se abandonó en modalidades de educación flexibles o el estudio de la educación media superior o superior a distancia o en modalidades semi-escolarizadas. Sin embargo, esto no siempre ocurre o bien los programas no son tan flexibles como lo demandan los compromisos familiares y laborales. De manera que las políticas públicas sectoriales (de educación en sus distintos niveles y trabajo, por ejemplo) pueden tender a re-normalizar las transiciones de los jóvenes (Du Bois-Reymond y López Blasco, 2004).

En particular, sobre las consecuencias del regreso a la escuela existen resultados mixtos. Se han hallado efectos positivos en el ingreso y el prestigio ocupacional (Felmlee, 1988; Light, 1995) y en el desarrollo personal (Bradburn, Moen y Dempster-McClain, 1995). El regreso a la escuela puede brindar a los individuos la oportunidad de obtener un mejor puesto de trabajo, así como de realizar la meta de completar niveles de estudio esperados. Sin embargo, también se han encontrado efectos negativos de corto plazo en la calidad de vida familiar de las madres que regresan a la escuela (Hostetler, Sweet y Moen, 2007) y en el nivel de estrés de los estudiantes en el área familiar y laboral (Kirby, Biever, Martinez y Gómez, 2004). 
El retorno escolar puede estar ligado a problemas de compatibilidad entre las trayectorias familiares y laborales, lo cual define el "costo" de retornar a la escuela o la oportunidad que tienen sólo ciertos individuos de poder hacerlo y de salir adelante con las demandas escolares (Astone, Shoen, Ensminger y Rothert, 2000). Estudiar puede resultar complicado al tener que lidiar con las responsabilidades que implica la participación laboral, el matrimonio o la crianza de los hijos (Hostetler, Sweet y Moen, 2007; Hostetler, 2008), siendo por ello importante el contexto de las circunstancias y los compromisos adquiridos para la decisión de regresar a la escuela.

En especial, los roles familiares pueden ser inhibidores del regreso a la escuela, ya que pueden demandar tiempo y energía que no pueden ser invertidos en otras actividades. Por ejemplo, un mayor número de hijos puede inhibir el regreso a la escuela al afianzar los roles de género tradicionales y la división del trabajo en el hogar, requiriendo que las mujeres se dediquen al hogar y los hombres que permanezcan en trabajos de tiempo completo para solventar los gastos (Bradburn, Moen y Dempster-McClain, 1995).

Otra variable asociada al regreso a la escuela es la etapa de crianza de los hijos, ya que los hijos demandan más atención y gastos de cuidados cuando son pequeños y, por lo tanto, esto puede tener un efecto negativo en regresar a la escuela (Felmlee, 1988). Sin embargo, estos resultados no se han sostenido en ciertos casos, como el de los hogares de doble ingreso, donde tener hijos pequeños en las mujeres tiene un efecto positivo sobre regresar a la universidad, tal vez porque ir a la universidad resulta menos demandante que un empleo de tiempo completo y tener hijos pequeños puede ser una oportunidad para dejar el trabajo y aumentar las credenciales educativas (Hostetler, Sweet y Moen, 2007; Carr y Sheridan, 2001).

Además de los roles adquiridos en el curso de vida, el retorno también es afectado por la previsión de la utilidad de esta acción, ya que la propensión a retornar puede variar dependiendo de los beneficios que pueden obtenerse (Astone, Shoen, Ensminger y Rothert, 2000; Hostetler, Sweet y Moen, 2007). Así, el regreso a la escuela podría estar motivado por aspiraciones de superación personal, ya sea porque se ha truncado un nivel de estudios o no se ha llegado al nivel de educación deseado (Hostetler, 2008).

Una variable crucial para la utilidad del retorno a la escuela es el nivel de estudios alcanzado. De acuerdo a la teoría del capital humano (Becker, 1993), estudiar tiene costos directos e indirectos. De manera que además de los costos directos de materiales, colegiaturas y manutención, están los 
costos indirectos o de oportunidad que son los ingresos que el individuo deja de percibir al estudiar. Así, el rendimiento esperado en el mercado de trabajo tiene que ser superior a la pérdida de ingreso mientras se estudia. Bajo esta lógica, estudios previos apoyan la evidencia de que quienes regresan a escuela son quienes más tienen que ganar al adquirir educación, siendo más probable que regresen a la escuela aquellos con mayores niveles escolares (Bradburn, Moen y Dempster-McClain, 1995; Astone, Shoen, Ensminger y Rothert, 2000).

También el retorno escolar podría indicar la necesidad de mayores credenciales o habilidades para el empleo, ya sea porque las habilidades para ciertos empleos se han hecho obsoletas y los mismos empleadores motivan la asistencia escolar o porque las condiciones de ciertos empleos son tan adversas que orillan a los trabajadores a un cambio de carrera laboral con mayores grados de calificación (Hostetler, Sweet y Moen, 2007). En el mejor de los casos, con el retorno escolar puede conseguirse una capacitación adecuada y, ante circunstancias favorables del mercado laboral, lograrse carreras laborales exitosas con una mayor remuneración económica.

$\mathrm{Al}$ respecto, una variable laboral que ha sido explorada en diversos estudios por su impacto positivo sobre el retorno a la escuela es la baja intensidad del trabajo. Es más probable que un individuo regrese a la escuela cuando se tiene un trabajo de tiempo parcial, que cuando se tiene uno de tiempo completo o se está fuera de la fuerza laboral, debido tanto al tiempo disponible para estudiar como a los ingresos que pueden ayudar a financiar la escuela (Bradburn, Moen y Dempster-McClain, 1995; Astone, Shoen, Ensminger y Rothert, 2000). Sin embargo, los resultados no son contundentes; también se ha encontrado una asociación directa entre regresar a la escuela y trabajar más de tiempo completo (Hostetler, Sweet y Moen, 2007).

En cuanto a la asociación de las condiciones laborales con el retorno a la escuela, se ha encontrado que las mujeres que tenían trabajos administrativos antes de que naciera su primer hijo tienden más a regresar a la escuela que las mujeres que nunca trabajaron o que tenían otras ocupaciones (Bradburn, Moen y Dempster-McClain, 1995). Otras características asociadas en Estados Unidos al retorno a la escuela han sido estar percibiendo bajos salarios y estar colocado en un empleo de bajo prestigio (Felmlee, 1988), así como tener un trabajo que no corresponde a las habilidades adquiridas en la escuela o con el que no se está satisfecho en términos de remuneración, oportunidades de movilidad o flexibilidad laboral (Hostetler, 2008). 
En México, es importante notar que son pocos los jóvenes que siguen la trayectoria lineal hacia a la adultez, es decir, que después de terminar la educación formal, ingresan a la fuerza laboral y, finalmente, forman su propia familia. Coubès y Zenteno (2005) encontraron que si bien esta es la trayectoria más común entre los hombres y las mujeres mexicanos de la cohorte 1966-68, sólo 44 por ciento de los jóvenes y 29 por ciento de las jóvenes la experimentaron, pues existen una variedad de trayectorias alternativas. Al comparar las trayectorias entre generaciones, los autores hallaron que, entre los hombres, este patrón no mostró crecimiento, mientras que entre las mujeres, el patrón normativo aumentó entre las generaciones 1936-38, 1951-53 y 1966-68, por la creciente participación de las mujeres en el mundo laboral.

En las mujeres, a la par que aumentó el patrón normativo, se incrementó la trayectoria de regreso a la escuela, después de haber trabajado, contraído matrimonio o tenido hijos. Pérez y Lindstrom (2014) encontraron que, de las generaciones antes mencionadas, alrededor de 1 de cada 10 individuos regresaron a la escuela, siendo mayor el retorno en las mujeres jóvenes. Los autores hallaron que mientras en la primera generación los hombres eran más propensos a regresar a la escuela que las mujeres, en la última generación el patrón se invirtió, correspondiendo al aumento de la escolarización y la participación laboral femenina. Este patrón es una muestra del cambio social experimentado en México ligado a las expectativas sociales sobre los roles de género apropiados, así como las crecientes oportunidades educativas y laborales para las mujeres.

Pérez y Lindstrom (2014) también mostraron que el retorno escolar se asoció en México a una serie de características personales y familiares de las poblaciones de las generaciones antes mencionadas como una menor edad, mayores niveles educativos, mayor nivel socioeconómico, no estar unido y tener antecedentes migratorios. La mayoría de estas variables indicaban que el retorno escolar es una decisión que implica una evaluación de los costos y los beneficios a obtenerse con la educación.

En resumen, desde la perspectiva de curso de vida, el regreso a la escuela ha sido conceptualizado como una transición que ocurre debido a las experiencias previas y los antecedentes socioeconómicos de los sujetos, así como las motivaciones individuales, los costos y la utilidad percibida de esta inversión en capital humano y las oportunidades del contexto social en cierto momento histórico (Bradburn, Moen y Dempster-McClain, 1995; Astone, Shoen, Ensminger y Rothert, 2000; Hostetler, 2008). En el caso de este artículo, las frecuencias de retornar a la escuela de las tres generacio- 
nes estudiadas corresponden, en tiempo histórico, de finales de los años 50 al 2011, año de levantamiento de la encuesta utilizada (EDER).

\section{Metodología}

\section{Fuente de información y métodos}

La fuente de información es la EDER 2011 (INEGI, El COLEF y UABC, 2013), la cual es una encuesta representativa de las áreas urbanas de México. Esta encuesta se levantó como un módulo anexo a la Encuesta Nacional de Ocupación y Empleo (ENOE) en las 32 áreas urbanas y metropolitanas auto-representadas, que constituyen 86 por ciento de las áreas urbanas del país y, por lo tanto, mantiene el mismo diseño muestral probabilístico, estratificado y por conglomerados. La EDER 2011 consta de 2,840 cuestionarios retrospectivos completos de individuos de las cohortes de nacimiento 1951-53, 1966-68 y 1978-80. Esta fuente de información contiene trayectorias completas sobre educación, trabajo, migración, nacimientos y uniones, edad por edad a partir de los seis años.

Este estudio se basa en una submuestra de 2,721 individuos con información en las variables de interés antes de los 33 años. Se incluyó solo información de estas edades para poder comparar las transiciones al retorno solamente durante la juventud de las tres generaciones (y no sobrestimar la exposición al riesgo de retorno de las generaciones de mayor edad).

Para lograr los objetivos planteados se empleó análisis de supervivencia. Se utilizaron tablas de vida con corrección para casos truncados, es decir, contando los episodios de exposición al riesgo que quedaron abiertos, como una herramienta para describir la temporalidad del retorno escolar. Luego, empleamos frecuencias para identificar el perfil de quienes regresaron a la escuela de acuerdo con las variables independientes que se detallan en el siguiente apartado y generamos tres modelos logísticos de riesgo discreto para analizar los condicionantes del regreso a la escuela (Rabe-Hesketh y Skrondal, 2008). Se estimaron modelos por separado para cada una de las variables explicativas ligadas al tipo de empleo, ya que guardaban una fuerte correlación. Por último, se usaron matrices de movilidad para describir los cambios ocurridos en el nivel de estudios, el tipo de ocupación y el sector de actividad, tras el periodo del retorno escolar.

\section{Variables de análisis}

En los modelos, la variable dependiente fue el regreso a la escuela, después de al menos un año fuera de la escuela. En total, se obtuvieron 603 
eventos de retorno escolar de 46,923 años persona en riesgo de regresar a la escuela, que resultaron de los 2,721 individuos analizados. Los episodios fuera de la escuela se construyeron a partir de los años-persona en que el individuo estaba expuesto al riesgo de regresar, considerando el riesgo a partir del año subsecuente del año de salida de la escuela, ya fuera por deserción escolar o el término de algún nivel. Se consideraron episodios cerrados aquellos que terminaron en retorno y episodios abiertos aquellos que sufrieron truncamiento por salir de observación de la encuesta o llegar a los 33 años.

La primera variable explicativa fue la etapa de crianza de los hijos, clasificada de acuerdo con las edades de los hijos por las demandas que pueden implicar, dado su propio grado de participación escolar: de 0-2 años, previo al preescolar, de 3-5 años, edades del preescolar, y de seis años en adelante, edades de educación básica en adelante. Se consideraron los 12 y 15 años de edad de los hijos como otros dos puntos claves para el reingreso a la escuela de los padres, por los otros niveles escolares y la posibilidad de mayor independencia de los hijos, pero ya no se presentaron diferencias.

En cuanto a las variables explicativas de las condiciones de empleo, se incluyeron la intensidad del empleo, el sector de actividad y el tipo de ocupación (no manual/manual) correspondientes al año previo del retorno escolar. La intensidad de empleo se definió a través de la jornada laboral, si era de tiempo parcial o de tiempo completo o más (40 horas o más por semana). Para el sector de actividad se empleó la clasificación elaborada por Coubès (2005), que permite identificar si el sector de actividad es formal o informal, a partir de las variables disponibles en la encuesta EDER (la rama de actividad y el número de trabajadores en el negocio o empresa). ${ }^{3}$ La clasificación contempla como parte del sector formal tanto al empleo público (en áreas de administración pública, educación y salud) como al empleo en medianas o grandes empresas (que emplean más de cinco personas en el sector terciario y más de 15 personas en la industria). Además, como parte del sector informal, la clasificación distingue entre el empleo agrícola (cualquier actividad en la agricultura, pesca o silvicultura) y la micro o pequeña empresa, que serían todas las actividades del sector terciario e industrial que no entran en la categoría de medianas o grandes empresas.

Para el tipo de ocupación se utilizó la clasificación de Pacheco (2005), que permite diferenciar las ocupaciones manuales de las no manuales. ${ }^{4}$

\footnotetext{
${ }^{3}$ La EDER no incluye otras variables para evaluar la informalidad en el empleo, más allá del sector, como las prestaciones laborales o la existencia de contratos.

${ }^{4}$ No se distinguió el grado de calificación dentro de las ocupaciones manuales y no manuales, porque en las ocupaciones no manuales tal grado de desagregación no era posible debido al tama-
} 
Como trabajadores en ocupaciones manuales se consideró a los trabajadores en actividades agropecuarias, artesanos y trabajadores fabriles, operadores de maquinaria fija en la industria, ayudantes, peones y similares, trabajadores ambulantes, en servicios personales y en servicios domésticos, además de despachadores y "checadores" en el transporte. Los trabajadores en ocupaciones no manuales fueron aquellos que, por corolario, no entraron en la categoría anterior.

Como variables control se incluyeron el sexo, la cohorte, la edad, la escolaridad de la madre ${ }^{5}$ y el tamaño de la localidad de residencia a la edad del retorno. El tamaño de la localidad de residencia es una variable cambiante en el tiempo, que brinda la encuesta para conocer si la localidad es urbana (de 15 mil habitantes y más) o rural (menor de 15 mil habitantes) en cada año calendario de cada entrevistado. También se consideraron los antecedentes de migración del individuo, el nivel estudios alcanzado y el estado civil, todas estas variables se tomaron de un año previo al retorno escolar (es decir, se desfasaron un año para incluirse en el modelo). Cabe señalar que la variable de migración incluye cambios de localidad hasta el año previo al regreso a la escuela, ya sea migración interna, internacional o ambos tipos. Suponemos que las oportunidades y los incentivos ligados al regreso a la escuela son diferentes de acuerdo con estas características, como se ha encontrado en estudios previos (Bradburn, Moen y DempsterMcClain, 1995; Hostetler, 2008; Pérez y Lindstrom, 2014).

Se probaron las interacciones con el sexo y la cohorte de todas las variables explicativas, pensando que pudiese haber una diferenciación por sexo por la división por sexo del trabajo en los hogares $\mathrm{y}$, por cohorte, por las diferencias históricas en los mercados laborales y la expansión educativa, pero no resultaron significativas (no mejoraron la bondad de ajuste del modelo). En cambio, la interacción de la cohorte con el sexo fue significativa $\mathrm{y}$, por lo tanto, se incluyó en los modelos que se presentan.

ño de muestra de los retornos escolares. Además, independientemente del grado de calificación, las ocupaciones manuales mostraron el mismo comportamiento relativo al retorno escolar, por lo que se decidió dejarlas en una sola categoría.

${ }^{5}$ Se toma en consideración solo la escolaridad de la madre, ya que la escolaridad del padre fue en menor medida especificada en la encuesta. Además, es reconocida la mayor influencia de la madre en las aspiraciones educativas y el capital cultural y socioeconómico de los hijos en el hogar y, por lo tanto, en su progresión en grados (Haveman y Wolfe, 1993; Hausmann and Szekely, 2003). 


\section{Resultados}

\section{Las tendencias y los perfiles de la población que regresa a la escuela}

En total, 18 por ciento de la población de las tres generaciones estudiadas (1951-53, 1966-68 y 1978-80) retornó a la escuela al menos una vez (Tabla 1). Este porcentaje es mucho mayor a diez por ciento registrado en conjunto en las generaciones 1936-38, 1951-53 y 1966-68 (Pérez y Lindstrom, 2014). Entre los hombres este porcentaje pasó de 18.2 por ciento en la primera generación, a 17 por ciento en la segunda y a 20.2 por ciento en la última, mientras en las mujeres aumentó gradualmente de 13.3 por ciento en la primera generación, a 16.1 por ciento en la segunda y a 21.2 por ciento en la tercera. Es decir, el regreso a la escuela en las mujeres fue mayor al paso del tiempo (desde los años 60), mientras que en los hombres esta frecuencia se contrajo ligeramente en la segunda generación para luego elevarse un poco en la tercera generación. En la primera generación los varones regresaron más a la escuela que las mujeres, pero esta diferencia se disipó al paso de las generaciones y en la generación más reciente las mujeres presentaron una mayor frecuencia de retorno escolar. Esto sugiere que al paso del tiempo entre las mujeres existieron menos barreras estructurales para la escolarización, lo cual podría estar ligado a cambios en las normas sociales de género y a los crecientes incentivos relativos a la educación formal en el mundo laboral.

Tabla 1: Porcentaje de la población con retorno escolar antes de los 33 años por número de retornos, sexo y generación. México, 2011

\begin{tabular}{lrrrrrrr}
\hline & \multicolumn{3}{c}{ Hombres } & \multicolumn{3}{c}{ Mujeres } \\
\hline \multirow{2}{*}{ Retorno escolar } & 1951 & 1966 & 1978 & 1951 & 1966 & 1978 & \multirow{2}{*}{ Total } \\
& 1953 & 1968 & 1980 & 1953 & 1968 & 1980 & \\
\hline \multirow{2}{*}{ Ninguno } & 81.8 & 83.0 & 79.8 & 86.7 & 83.9 & 78.8 & 81.9 \\
Una vez & 12.9 & 15.3 & 16.6 & 10.1 & 13.0 & 17.4 & 14.8 \\
Dos o más & 5.3 & 1.6 & 3.6 & 3.2 & 3.2 & 3.8 & 3.3 \\
Total & 100.0 & 100.0 & 100.0 & 100.0 & 100.0 & 100.0 & 100.0 \\
Total al menos una vez & 18.2 & 17.0 & 20.2 & 13.3 & 16.1 & 21.2 & 18.1 \\
$\mathrm{n}$ & 418 & 422 & 502 & 413 & 442 & 524 & 2,721 \\
\hline
\end{tabular}

Fuente: cálculos propios con base en EDER, 2011.

Al evaluar los episodios fuera de la escuela y el evento de reingreso escolar dentro de los episodios (Tabla 2), se observa que, en las últimas dos generaciones, las mujeres también mostraron una mayor duración media de estar fuera de la escuela antes del retorno y una mayor edad media a la 
ocurrencia de este evento que los hombres. Asimismo, el rango intercuartil de las edades al retorno aumentó en las mujeres, mientras que en los hombres se mantuvo estable, llegando a ser para la última cohorte de 11 años (de los 16 a los 26 años) en las mujeres y de siete años (de los 16 a los 22 años) en los hombres. Es decir, la adquisición de capital humano en las mujeres ocurrió de forma más difusa que en los hombres, en una etapa más amplia del curso de vida; lo cual indica la complejidad del retorno escolar para las mujeres, posiblemente ante la dificultad de combinar las actividades educativas con las responsabilidades familiares, como se ha encontrado en estudios previos (Hostleter, Sweet y Moen, 2007).

Tabla 2: Características del retorno escolar de la población antes de los 33 por sexo y generación. México, 2011

\begin{tabular}{lrrrrrrr}
\hline & \multicolumn{3}{c}{ Hombres } & \multicolumn{5}{c}{ Mujeres } \\
\hline Retorno escolar & 1951 & 1966 & 1978 & 1951 & 1966 & 1978 & Total \\
\hline \% de retorno en & 1953 & 1968 & 1980 & 1953 & 1968 & 1980 & \\
episodios & 19.5 & 16.3 & 19.6 & 14.6 & 16.9 & 21.3 & 18.4 \\
Duración media & 3.9 & 3.8 & 3.5 & 3.5 & 4.7 & 4.1 & 4.0 \\
Edad media & 19.6 & 20.6 & 19.6 & 18.7 & 20.8 & 20.6 & 20.2 \\
Edad percentil 25 & 16 & 17 & 16 & 15 & 16 & 16 & 16.0 \\
Edad percentil 75 & 23 & 22 & 22 & 22 & 27 & 26 & 23.0 \\
n retornos & 104 & 81 & 113 & 77 & 87 & 141 & 603 \\
n episodios & 513 & 496 & 598 & 485 & 513 & 640 & 3,245 \\
\hline Frion
\end{tabular}

Fuente: cálculos propios con base en EDER, 2011.

Las funciones de supervivencia al retorno escolar (Figura 1), en los años posteriores al abandono de la escuela, indican que las brechas entre hombres y mujeres en el tiempo de retorno escolar eran muy notorias en la primera generación, que se redujeron en las generaciones subsecuentes. A diferencia de las primeras generaciones, en las que los hombres registraron un mayor retorno que las mujeres, en la última generación se observa una mayor probabilidad acumulada de haber regresado a la escuela en las mujeres que en los hombres, a partir de los 12 años de haber salido de la escuela, lo cual corrobora lo difusa que resulta ser la escolarización femenina.

En cuanto a las diferencias por cohorte en la función de supervivencia al retorno escolar (Figura 1), en las mujeres se observa en la última generación un aumento notorio del regreso a la escuela en los primeros 20 años de abandono escolar, en cambio en los hombres se registra un ligero incre- 
mento en la tercera generación, respecto a la primera generación, tras un retroceso en la segunda generación. La ligera reducción en el regreso a la escuela de la segunda generación de hombres pudo deberse al efecto de las continuas crisis económicas de los años 80 en las oportunidades educativas de los jóvenes. En esta época, los hogares tuvieron que aumentar el número de trabajadores, por lo tanto, más hijos varones salieron al mercado laboral para contribuir al ingreso de sus hijos, mientras que las mujeres apoyaron a sus madres en las tareas domésticas (García y Pacheco, 2000).

Figura 1: Función de supervivencia al retorno escolar por sexo y cohorte. México, 2011

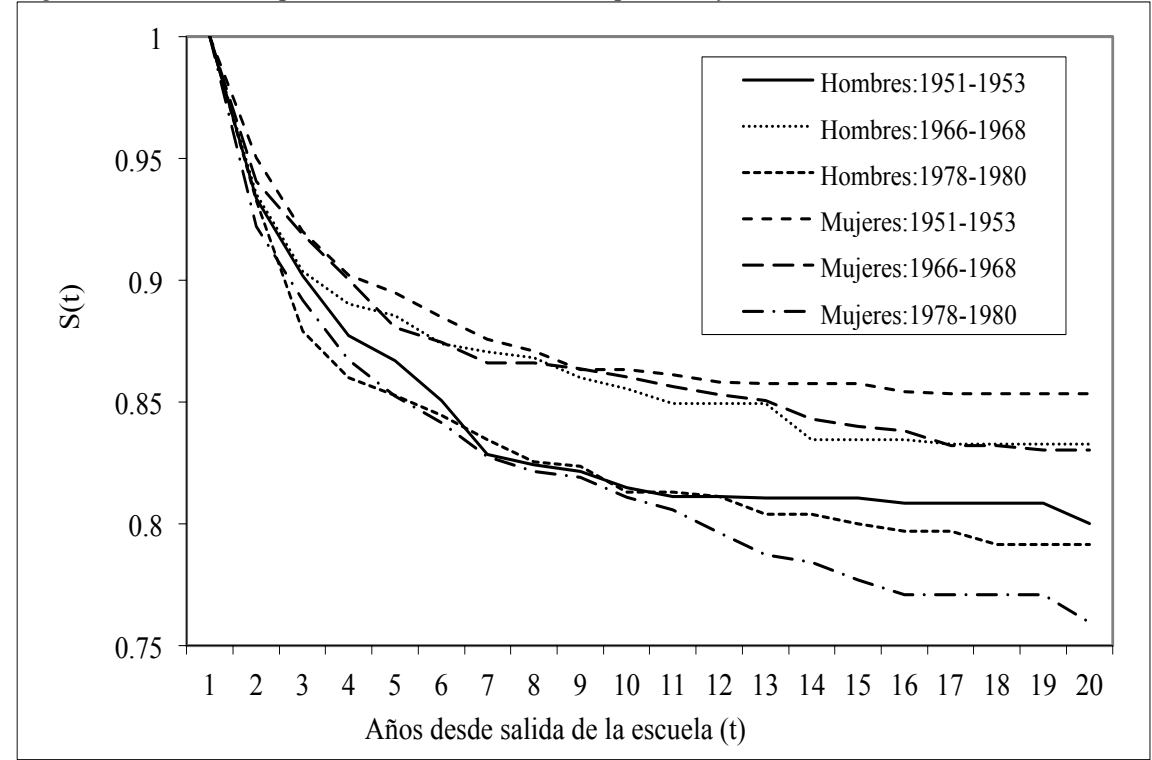

Fuente: cálculos propios con base en EDER, 2011.

En cuanto a los perfiles socioeconómicos de las poblaciones en riesgo de retornar a la escuela (Tabla 3), se encuentra que el retorno fue más frecuente a menor edad, en el nivel medio o medio superior, que en otros niveles educativos, cuando no se tenían hijos, durante la soltería y a mayor educación de la madre. Además, el regresar a la escuela fue más frecuente entre quienes no trabajan o trabajan menos de tiempo completo, entre quienes trabajaban en el sector público o en empleos no manuales. Estas frecuencias sugieren la existencia de relaciones estadísticas con el regreso a la escuela, para corroborarlo se estiman a continuación modelos multivariados. 


\section{Los condicionantes del retorno escolar y los logros obtenidos}

Los modelos logísticos multivariados se presentan en la Tabla 4. En cuanto a las variables explicativas, la etapa de crianza de los hijos fue determinante en el regreso a la escuela. El riesgo de regresar a la escuela fue 33 por ciento menor cuando los hijos estaban pequeños ( $0-2$ años) que cuando los individuos no tenían hijos, pero el riesgo de retornar se incrementó cuando los hijos tenían edad de cursar la educación primaria (seis o más años). En este caso, el riesgo de regresar a la escuela fue el doble que cuando no se tenían hijos. Es decir, a medida que las demandas parentales disminuyeron, la incorporación a la escuela de los jóvenes padres de familia se convirtió en una opción. Es importante aclarar que la etapa de crianza fue la variable que se asoció al retorno escolar y no solamente tener hijos o no, pues en la etapa en que los hijos están pequeños fue menor la propensión a regresar a la escuela, pero al llegar los hijos a edad escolar la propensión fue mayor que para quienes no tenían hijos.

Respecto a las variables laborales, no trabajar favoreció el retorno escolar, así como trabajar bajo ciertas condiciones. No hubo diferencias significativas entre tener un trabajo de tiempo parcial o no trabajar para el riesgo de regresar a la escuela, en cambio un trabajo de tiempo completo o más disminuyó el riesgo de retorno, siendo 35 por ciento menor si se tenía este tipo de empleo, que si no se tenía empleo. Esto nos puede indicar, por un lado, la dificultad que representa compatibilizar un empleo de tiempo completo y la escuela. Por otro lado, también nos sugiere que tener un empleo de tiempo parcial podría incentivar el regreso a la escuela para mejorar las oportunidades laborales y, por ello, ser parecido a no haber adquirido compromisos laborales.

Trabajar en el sector formal per se no se asoció al retorno escolar, como inicialmente se había planteado. Trabajar en el sector público dobló la posibilidad de reinsertarse en la escuela, respecto a trabajar en medianas o grandes empresas. En contraste, no hubo diferencias significativas en el riesgo de retornar de los trabajadores de micro o pequeñas empresas y del sector agrícola, respecto a la misma categoría de referencia.

El sector público ha fomentado desde los años 70, especialmente a partir de los años 80, así como en la última década, el retorno escolar de sus empleados a través del Instituto Nacional para la Educación de los Adultos (INEA) (SEP, 2009). Además, este retorno podría estar vinculado a la posibilidad de movilidad ocupacional asociada a la educación en este sector. Por ejemplo, algunos gremios como el de los trabajadores de la educación han impulsado la capacitación continua de los maestros o el personal escolar para el ascenso en escalafón. 
Tabla 3: Distribución porcentual de la población en riesgo de retorno escolar en el año del retorno y en los años de no retorno por características selectas. México, 2011

\begin{tabular}{|c|c|c|c|c|}
\hline Variable independiente & Categoría & Retorno & No retorno & Total \\
\hline \multirow[t]{4}{*}{ Etapa de crianza de los hijos } & Sin hijos & 85.6 & 46.8 & 47.3 \\
\hline & Algún hijo de 0-2 años & 7.3 & 30.2 & 29.9 \\
\hline & Algún hijo de 3-5 años & 4.3 & 13.8 & 13.7 \\
\hline & Algún hijo de 6 años o más & 2.8 & 9.2 & 9.1 \\
\hline \multirow[t]{3}{*}{ Condición de actividad (lag) } & Tiempo completo o más & 43.2 & 60.4 & 59.1 \\
\hline & Menor que tiempo completo & 7.4 & 5.9 & 5.9 \\
\hline & No trabajó & 49.4 & 33.7 & 35.0 \\
\hline \multirow[t]{4}{*}{ Sector de actividad (lag) } & Mediana y grande empresa & 41.5 & 42.0 & 42.0 \\
\hline & Agricultura & 4.8 & 3.9 & 3.9 \\
\hline & Micro y pequeña empresa & 32.2 & 39.9 & 39.8 \\
\hline & Sector público & 21.5 & 14.2 & 14.3 \\
\hline \multirow[t]{2}{*}{ Tipo de ocupación (lag) } & No manual & 64.5 & 52.4 & 52.6 \\
\hline & Manual & 35.5 & 47.6 & 47.4 \\
\hline \multirow[t]{3}{*}{ Cohorte } & $1951-1953$ & 18.3 & 24.6 & 24.5 \\
\hline & 1966-1968 & 33.9 & 40.0 & 40.0 \\
\hline & $1978-1980$ & 47.8 & 35.4 & 35.5 \\
\hline \multirow[t]{2}{*}{ Sexo } & Hombres & 47.9 & 46.0 & 46.1 \\
\hline & Mujeres & 52.1 & 54.0 & 53.9 \\
\hline Edad & & 20.2 & 25.2 & 25.1 \\
\hline \multirow[t]{5}{*}{ Nivel de estudios (lag) } & Primaria & 31.1 & 37.8 & 37.7 \\
\hline & Secundaria & 31.3 & 28.3 & 28.3 \\
\hline & Preparatoria & 23.9 & 13.8 & 13.9 \\
\hline & Técnica o normal & 4.7 & 9.8 & 9.7 \\
\hline & Profesional & 9.0 & 10.3 & 10.3 \\
\hline \multirow[t]{2}{*}{ Migración previa (lag) } & No & 54.6 & 54.3 & 54.3 \\
\hline & Sí & 45.4 & 45.7 & 45.7 \\
\hline \multirow[t]{3}{*}{ Estado civil (lag) } & Casado o unido & 13.2 & 53.4 & 52.9 \\
\hline & Soltero & 85.0 & 43.0 & 43.5 \\
\hline & Separado o divorciado & 1.8 & 3.6 & 3.6 \\
\hline \multirow[t]{6}{*}{ Escolaridad de la madre } & Sin educación formal & 14.9 & 27.9 & 27.8 \\
\hline & $1-5$ años & 25.1 & 24.1 & 24.1 \\
\hline & 6-8 años & 29.8 & 27.1 & 27.2 \\
\hline & 9-11 años & 15.3 & 8.5 & 8.6 \\
\hline & 12 años o más & 10.0 & 6.2 & 6.2 \\
\hline & No especificado o sin madre & 4.9 & 6.2 & 6.2 \\
\hline \multirow[t]{3}{*}{ Tamaño de la localidad } & Rural & 9.2 & 8.5 & 8.5 \\
\hline & Urbano & 88.6 & 88.7 & 88.7 \\
\hline & No especificado u otro país & 2.2 & 2.7 & 2.7 \\
\hline $\mathrm{n}$ & & 603 & 46320 & 46923 \\
\hline
\end{tabular}

Fuente: cálculos propios con base en EDER, 2011. 
Tabla 4: Modelo logístico de tiempo discreto de regresar a la escuela antes de los 33 años. México, 2011

\begin{tabular}{|c|c|c|c|c|c|c|c|}
\hline \multirow[b]{2}{*}{ Variable independiente } & \multirow[b]{2}{*}{ Categoría } & \multicolumn{2}{|c|}{ Modelo 1} & \multicolumn{2}{|c|}{ Modelo 2} & \multicolumn{2}{|c|}{ Modelo 3} \\
\hline & & OR & $\mathrm{P}>|\mathrm{z}|$ & OR & $\mathrm{P}>|\mathrm{z}|$ & OR & $\mathrm{P}>|\mathrm{z}|$ \\
\hline \multirow{3}{*}{$\begin{array}{l}\text { Etapa de crianza de los hijos } \\
\text { (Sin hijos) }\end{array}$} & Algún hijo de 0-2 años & 0.67 & $*$ & 0.67 & $*$ & 0.67 & $*$ \\
\hline & Algún hijo de 3-5 años & 1.31 & & 1.31 & & 1.31 & \\
\hline & Algún hijo de 6 años o más & 2.11 & $*$ & 2.08 & $*$ & 2.10 & $*$ \\
\hline \multirow{6}{*}{$\begin{array}{l}\text { Condición de actividad-lag } \\
\text { (No trabajó) } \\
\text { Sector de empleo-lag } \\
\text { (Mediana y grande empresa) }\end{array}$} & Tiempo completo o más & 0.65 & $* * *$ & & & -- & \\
\hline & Menor a tiempo completo & 0.86 & & & & -- & \\
\hline & Agricultura & & & 1.13 & & -- & \\
\hline & Micro y pequeña empresa & & & 0.96 & & -- & \\
\hline & Sector público & & & 2.04 & $* * *$ & -- & \\
\hline & No trabajó & & & 1.60 & $* *$ & -- & \\
\hline \multirow{7}{*}{$\begin{array}{l}\text { Tipo de ocupación-lag } \\
\text { (No trabajó) } \\
\text { Cohorte* Sexo } \\
\text { (Mujeres 1951-1953) }\end{array}$} & No manual & & & & & 0.85 & \\
\hline & Manual & & & & & 0.53 & $* * *$ \\
\hline & Hombres 1951-1953 & 1.51 & $*$ & 1.56 & $*$ & 1.60 & $*$ \\
\hline & Mujeres 1966-1968 & 1.15 & & 1.15 & & 1.16 & \\
\hline & Hombres 1966-1968 & 1.08 & & 1.12 & & 1.14 & \\
\hline & Mujeres 1978-1980 & 1.36 & + & 1.41 & + & 1.39 & + \\
\hline & Hombres 1978-1980 & 1.18 & & 1.24 & & 1.24 & \\
\hline \multirow{5}{*}{$\begin{array}{l}\text { Edad } \\
\text { Nivel de estudios-lag } \\
\text { (Primaria) }\end{array}$} & & 0.84 & $* * *$ & 0.83 & $* * *$ & 0.84 & $* * *$ \\
\hline & Secundaria & 2.00 & $* * *$ & 2.02 & $* * *$ & 1.98 & $* * *$ \\
\hline & Preparatoria & 4.12 & $* * *$ & 4.04 & $* * *$ & 3.76 & $* * *$ \\
\hline & Técnica o normal & 1.20 & & 1.11 & & 1.11 & \\
\hline & $\begin{array}{l}\text { Profesional } \\
\text { (No) }\end{array}$ & 2.82 & $* * *$ & 2.49 & $* * *$ & 2.42 & $* * *$ \\
\hline Migración previa-lag & Sí & 1.56 & $* * *$ & 1.54 & $* * *$ & 1.54 & $* * *$ \\
\hline \multirow{7}{*}{$\begin{array}{l}\text { Estado civil-lag } \\
\text { (Casado o unido) } \\
\text { Escolaridad de la madre } \\
\text { (Sin educación formal) }\end{array}$} & Soltero & 2.92 & $* * *$ & 2.89 & $* * *$ & 2.89 & $* * *$ \\
\hline & Separado o divorciado & 2.11 & $*$ & 2.18 & $*$ & 2.08 & $*$ \\
\hline & 1-5 años & 1.86 & $* * *$ & 1.86 & $* * *$ & 1.85 & $* * *$ \\
\hline & 6-8 años & 1.67 & $* * *$ & 1.63 & $* *$ & 1.61 & $* *$ \\
\hline & 9-11 años & 2.55 & $* * *$ & 2.53 & $* * *$ & 2.48 & $* * *$ \\
\hline & 12 años o más & 3.17 & $* * *$ & 3.09 & $* * *$ & 3.04 & $* * *$ \\
\hline & No especificado o sin madre & 1.20 & & 1.19 & & 1.16 & \\
\hline \multirow{6}{*}{$\begin{array}{l}\text { Tamaño de la localidad } \\
\text { (Rural) } \\
\text { Episodio fuera de la escuela } \\
\text { (Primero) } \\
\text { Log-pseudolikelihood } \\
\text { Número de años persona }\end{array}$} & Urbano & 1.49 & $*$ & 1.53 & $*$ & 1.48 & $*$ \\
\hline & No especificado u otro país & 0.77 & & 0.80 & & 0.79 & \\
\hline & & & & & & & \\
\hline & Segundo o subsecuente & 2.55 & $* * *$ & 2.57 & $* * *$ & 2.53 & $* * *$ \\
\hline & & \multirow{2}{*}{\multicolumn{2}{|c|}{$\begin{array}{c}-14159757 \\
46923 \\
\end{array}$}} & \multirow{2}{*}{\multicolumn{2}{|c|}{$\begin{array}{c}-14109014 \\
46923 \\
\end{array}$}} & \multirow{2}{*}{\multicolumn{2}{|c|}{$\begin{array}{c}-14130882 \\
46923 \\
\end{array}$}} \\
\hline & & & & & & & \\
\hline
\end{tabular}

OR: Razón de momios

$* * * \mathrm{p}<0.001$

$* * \mathrm{p}<0.01$

$* \mathrm{p}<0.05$

$+\mathrm{p}<0.1$

Categoría de referencia en paréntesis

Fuente: cálculos propios con base en EDER, 2011.

De manera similar, en años recientes el Gobierno Federal a través del Consejo Nacional de Ciencia y Tecnología (CONACYT) creó el Programa de Formación de Alto Nivel para la Administración Pública Federal, el cual incentiva a servidores públicos con alto potencial a que regresen a la escuela a cursar estudios de posgrado, ya sea en México o en el extranjero, 
al otorgarles una serie de estímulos económicos atractivos mediante becas que cubren cuotas de inscripción y manutención (CONACYT, 2014).

En cuanto al resto de las variables independientes, los hombres tuvieron mayor propensión de retornar a la escuela en la primera generación que las mujeres, pero esta tendencia se revirtió en la última generación. En este sentido, el reingreso a la escuela de las mujeres más jóvenes se ha beneficiado de las políticas a favor de la educación de adultos y los cambios en las nociones sobre los roles de género. Además, a menor edad fue mayor el retorno escolar, es lógico pensar que entre menos tiempo haya pasado del abandono escolar y menos compromisos se hayan adquirido, más fácil es reinsertarse en la escuela.

También se confirmó que el regreso a la escuela fue más frecuente en el nivel medio superior, seguido por el superior y por el medio, a comparación de la primaria. Esto podría vincularse a las tasas más altas de deserción en estos niveles, sobre todo al concluir el nivel medio y comenzar la educación superior (Brunet, 2016), así como a la expansión de la obligatoriedad del nivel medio superior (SEGOB, 2012), que hizo la preparatoria necesaria para la inserción en muchos empleos del sector formal.

Otra característica asociada al reingreso a la escuela fue el haber migrado en el transcurso de la vida, al controlar el efecto de las otras variables independientes. Si bien la migración interna e internacional tienen un efecto directo en el abandono y la interrupción escolar e indirecto en el logro educativo en México (Vargas y Potter, 2011), la migración y la escolarización podrían ser complementarias en las ciudades. Esto por las características socioeconómicas selectivas de los migrantes, sus motivaciones educativas, que podrían estar ligadas a la búsqueda de mejores oportunidades laborales, o por la intersección de estas motivaciones con las oportunidades que ofrecen las ciudades de recepción (Peinador, 2005).

Las variables asociadas al entorno y el origen social de los jóvenes se vincularon a regresar a la escuela, lo cual apoya la amplia evidencia en países en desarrollo sobre la asociación entre la mayor escolarización, a mayor estatus socioeconómico y oportunidades educativas (Buchmann y Hannum, 2001). Vivir en zonas urbanas y tener madres con alta escolaridad, mayor a los nueve años, se ligaron a un mayor retorno escolar. En las ciudades, se ha tenido mayor y mejor infraestructura escolar, además de que en ellas el valor por la educación se incrementa debido a las oportunidades que brinda el mercado laboral. Además, una más alta escolaridad de los padres está ligada no solo a un mayor nivel socioeconómico sino a un mayor capital cultural en el hogar, el cual beneficia el aprovechamiento y el logro escolar 
de los hijos (Farkas, 1996). Una mayor escolaridad materna no solo influye positivamente en las aspiraciones educativas de los hijos, por el rol que juegan las madres en el proceso de socialización de los hijos, sino también en el nivel de ingreso de los hogares y las oportunidades escolares de los hijos (Hausmann y Szekely, 2003).

El análisis multivariado también corroboró que el regreso a la escuela se dio con mayor frecuencia cuando no se tenía pareja o habían ocurrido eventos vitales asociados a la disolución conyugal como estar separado o divorciado, respecto a estar unido. En estudios previos se ha probado que el abandono escolar se asocia directamente con dejar de corresidir con los padres y adquirir roles conyugales (Rabell y Murillo, 2016; Brunet, 2016). Así también la posibilidad de reingresar a la escuela es mayor cuando no se han adquirido roles conyugales o se deja de tenerlos.

Por último, para evaluar las posibilidades de movilidad educativa o laboral de los jóvenes que retornaron a la escuela se crearon matrices del nivel educativo, el sector de actividad y el tipo de ocupación antes y después del retorno en los episodios cerrados, es decir, aquellos episodios que terminaron con otra salida de la escuela (Tabla 5).

En primer lugar, se observó una importante movilidad educativa, la cual fue mayor en quienes el regreso a la escuela ocurrió durante la secundaria (Panel A de la Tabla 5), 79 por ciento alcanzaron un nivel más alto. Les siguieron quienes retornaron en la primaria ( 73 por ciento lograron un nivel mayor) y, finalmente, quienes retornaron en el nivel de educación técnica o normal (65 por ciento alcanzaron un grado superior). Entre quienes retornaron en el nivel medio superior, 10.3 por ciento alcanzaron la universidad, ya se encontraban más avanzados en sus estudios. Estos resultados evidencian que los efectos de regresar a la escuela en la trayectoria educativa de los jóvenes son positivos.

La movilidad en el sector de actividad después del episodio de retorno escolar también apoya la importancia de esta inversión en capital humano. Entre quienes no trabajaban antes del retorno escolar, la mitad continuó sin trabajar, un cuarto terminó empleándose en empresas mediana o grande y alrededor de ocho por ciento en el sector público. Entre quienes trabajaban en el sector informal (micro o pequeña empresa o agricultura) ${ }^{6}$ cerca de 30 por ciento pasó a medianas o grandes empresas y otro tres por ciento al sector público. En contraste, el cambio de algún trabajador de los sectores de empleo formal a alguno de los sectores de empleo informal o fuera del empleo ocurrió con menor frecuencia.

\footnotetext{
${ }^{6}$ Se unieron ambos sectores, ya que el tamaño de la muestra en agricultura era muy reducido.
} 
La reinserción en la escuela y su vinculación con la vida familiar y laboral de tres ... /E.D. VARGAS y P.P. ORRACA

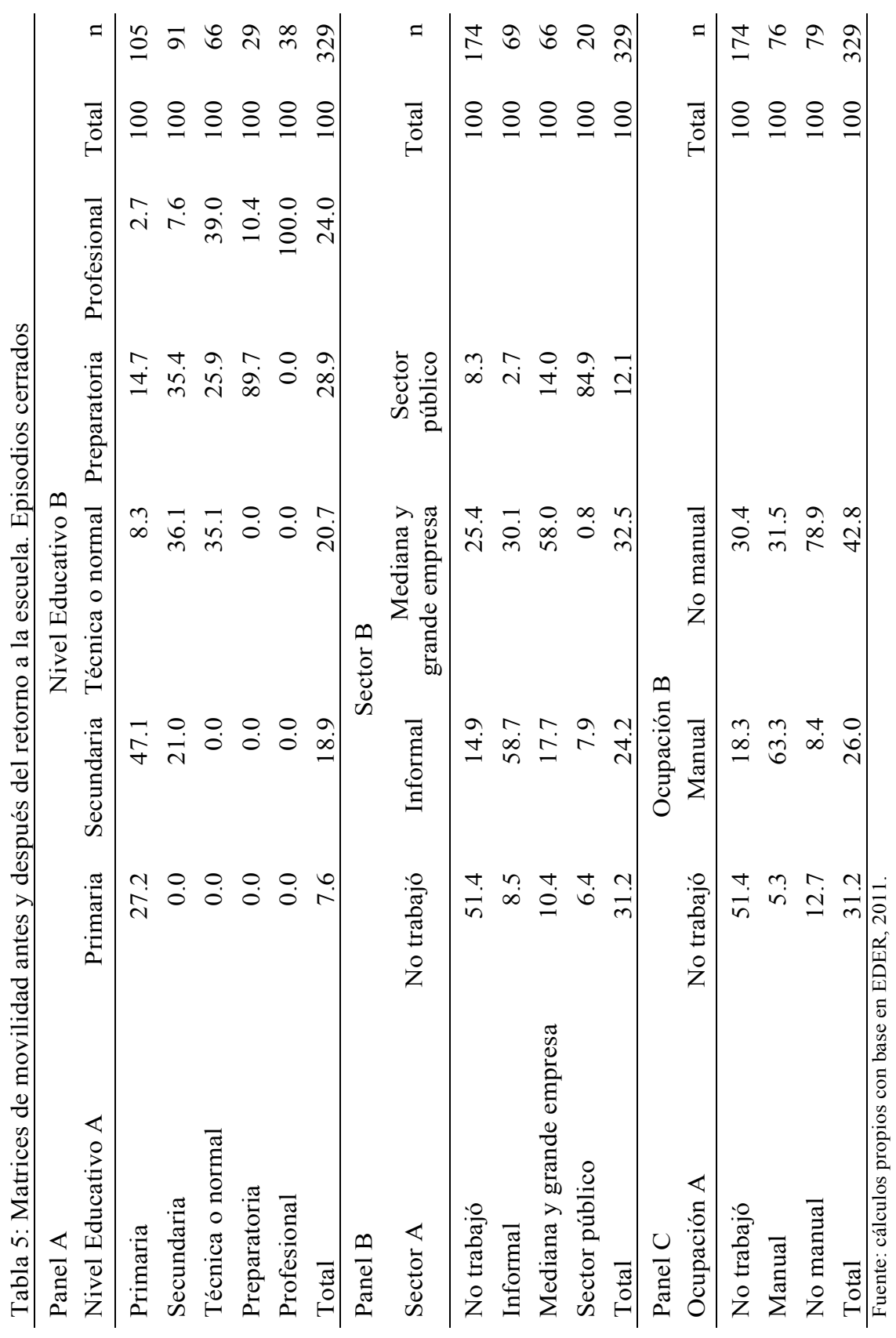


En cuanto a la movilidad de acuerdo al tipo de ocupación, fue mayor la movilidad en tiempo B de quienes se encontraban en empleos manuales en tiempo A que entre quienes realizaban empleos no manuales. Es decir, fue mayor la movilidad ascendente, 31.5 por ciento de quienes se encontraban en empleos manuales pasaron a empleos no manuales. En cambio, solo 8.4 por ciento de quienes se encontraban en empleos no manuales pasaron a empleos manuales.

\section{Conclusiones}

Los resultados de este trabajo muestran la importancia del retorno escolar en las trayectorias de vida de tres generaciones de mexicanos. En ambos sexos, el retorno fue ligeramente más común en la cohorte 1978-1980 que en las cohortes precedentes, aproximadamente uno de cada cinco mexicanos retornó a la escuela alguna vez en la vida. Las campañas a favor de educación de los adultos desde finales de los años 70, los cambios a favor de la equidad de género en la educación, las demandas de trabajadores más calificados en ciertos sectores del mercado laboral y la expansión de la educación básica son procesos sociales que pueden vincularse a esta tendencia.

Se registraron diferencias por sexo, siendo el incremento del regreso a la escuela mayor en las mujeres. Brunet (2016) encontró que las mujeres de las generaciones 1966-1968 y 1978-1980, respecto a la generación 1951-53, tuvieron mayores oportunidades de permanecer en la escuela; pero este aumento en la retención escolar no fue significativo en los hombres. De la misma manera, esta investigación arroja que son las mujeres de la cohorte 1978-1980 quienes amplían significativamente sus posibilidades de reingresar al sistema educativo, pero no los varones, quienes ya desde la primera generación analizada mostraban altas frecuencias de reingreso escolar.

Es importante notar que el retorno a la escuela se registró en mayor medida en los niveles educativos que han representado el "cuello" de botella para la continuidad escolar, es decir, donde las probabilidades de deserción también son más elevadas como es el nivel medio superior. Además, las desigualdades de origen y el contexto socioeconómico jugaron un papel esencial en las oportunidades de los jóvenes de regresar a la escuela. Los jóvenes con mayor capital humano y cultural en el hogar y residentes de las ciudades fueron quienes tuvieron mayores propensiones de regresar a la escuela. En este sentido, queda claro que las oportunidades para regresar a la escuela son mayores para quienes ocupan lugares más altos en la escala 
social. Los resultados corroboran que los más favorecidos de los desfavorecidos son quienes logran regresar a la escuela (Hostleter, 2008).

En cuanto a los factores asociados al reingreso a la escuela, este estudio muestra que efectivamente las trayectorias educativa, laboral y familiar se encuentran entrelazadas y es en el contexto de estas intersecciones que los individuos optan por regresar a la escuela. Así, esta transición es una muestra de la interdependencia de las distintas esferas de actividad del ser humano, así como de la agencia humana en respuesta a los cambios sociales que impulsan la adquisición de capital humano (Bradburn, Moen y Dempster-McClain, 1995; Hostetler, 2008). Tanto la etapa de crianza de los hijos como las condiciones de trabajo fueron importantes en las propensiones de regresar a la escuela. Tal como esperábamos, los mexicanos reingresaron a la escuela en el momento cuando se pudieron compatibilizar los roles de estudiante con otros roles como el de trabajador y ser padres, $o$ bien cuando no se habían adquirido estos roles. Las demandas de tiempo y los costos de otras actividades familiares y laborales obstruyen de manera significativa la reinserción a la escuela.

Los resultados confirman, por un lado, que no es el hecho de tener hijos lo que actúa en detrimento de regresar a la escuela, sino las obligaciones familiares vinculadas a la crianza de hijos pequeños, las cuales pueden competir con el tiempo y los recursos que se pueden dedicar a la escuela. Por otro lado, también se encuentra que la alta intensidad del trabajo obstaculiza la incorporación a la escuela. A pesar de que en otros contextos, el empleo de tiempo parcial se asocia en mayor medida al regreso a la escuela que estar sin empleo (Bradburn, Moen y Dempster-McClain, 1995; Astone, Shoen, Ensminger y Rothert, 2000), por la necesidad de financiamiento para la continuidad escolar, en México no hubo diferencias significativas entre no trabajar y tener un empleo de tiempo parcial para la reincorporación a la escuela. Esto puede estar vinculado al papel que juegan las escuelas y los programas educativos públicos en nuestro país, en especial de nivel superior, que son de bajo costo, mientras que en otros países están altamente privatizados.

Otro hallazgo fue que el regreso a la escuela ocurre cuando las condiciones del empleo incentivan esta inversión. Dentro de los desertores, los trabajadores que tuvieron mayor propensión de retornar a la escuela fueron aquellos en empleos no manuales y en el sector público. Esto confirmó parcialmente la hipótesis inicial, pues planteamos que los trabajadores con mayores oportunidades por parte de los empleadores y mayores motivaciones de movilidad laboral serían quienes regresarían a la escuela, inclu- 
yendo en este perfil a todos los trabajadores formales y no manuales. Sin embargo, los resultados mostraron que los trabajadores de las empresas mediana y grande no necesariamente tuvieron las mismas oportunidades que los empleados del sector público, a pesar de estar en el sector formal. La industria, por ejemplo, que emplea grandes contingentes de trabajadores, ofrece trabajos intensivos que difícilmente son compatibles con la educación formal (Hernández y Vargas, 2016).

No obstante, en las matrices de movilidad, se observaron claros beneficios laborales en aquellos provenientes de estos sectores menos privilegiados. En general, la movilidad laboral ascendente fue mucho mayor que la descendiente en términos laborales entre todos aquellos que experimentaron el retorno escolar. Asimismo, se registró una importante movilidad de nivel educativo, lo cual apoya la continuidad y el fomento de programas de educación para adultos y trabajadores que sean lo suficientemente flexibles, atiendan a la población que sigue trayectorias escolares fragmentadas y heterogéneas y contribuyan a la capitación para el trabajo y la movilidad ocupacional de los mexicanos.

Los hallazgos de este estudio muestran que el regreso a la escuela fue mayor con el paso del tiempo, lo cual puede ser atribuido parcialmente a las políticas educativas encaminadas a este propósito. Sin embargo, aún observamos una fuerte desigualdad para el reingreso al mundo escolar de aquellos jóvenes con hijos pequeños, con jornadas de alta intensidad, en empleos manuales y en sectores distintos al público. Dado que la sociedad ya no provee las garantías de empleo y protección social del pasado, el estado podría influir aún más en el re-ingreso a la escuela de los jóvenes, brindando oportunidades de guarderías con educación inicial para los hijos de madres estudiantes, así como creando alianzas con las empresas privadas para la contratación de estudiantes y la creación de empleos con horarios flexibles. Esto con el fin de aminorar la incertidumbre de aquellos jóvenes que experimentan modelos no lineales de transición a la vida adulta y quieren hacer mayores inversiones en educación siendo trabajadores y padres de familia.

\section{REFERENCIAS BIBLIOGRÁFICAS}

Astone, Marie; Schoen, Robert; Ensminger, Margaret y Rothert, Kendra, 2000, "School Reentry in Early Adulthood: The Case of Inner-City African Americans", en Sociology of Education, 73(3): 133-154.

Becker, Gary, 1993, Human Capital: A Theoretical and empirical analysis with especial reference to education, The University of Chicago Press, Chicago. 
Bradburn, Ellen M.; Moen, Phyllis y Dempster-McClain, Donna, 1995, "Women's return to school following the transition to motherhood", en Social Forces, 73: $1517-1551$.

Brunet, Nicolás, 2016, "Dejar la escuela en perspectiva longitudinal micro-macro: marcas biográficas y contextuales", en Coubès, Marie Laure, Solís, Patricio, y Zavala de Cosío, María E. (coords.) Generaciones, cursos de vida y desigualdad social en México, El Colegio de México, El Colegio de la Frontera Norte, México.

Buchmann, Claudia y Hannum, Emily, 2001, "Education and Stratification in Developing Countries: A Review of Theories and Research", en The Annual Review of Sociology, 27: 77-102.

Carr, Deborah y Sheridan, Jennifer, 2001, "Family turning-points and career transitions at midlife", en Marshall, Victor, Heinz, Walter, Kruger, Helga y Verma, Anil (eds.) Restructuring work and the life course, University of Toronto Press, Toronto.

CONACYT, 2014, Formación para la Administración Pública, México, Consejo Nacional de Ciencia y Tecnología (Consultado 20/septiembre/2017). Disponible en www.conacyt.gob.mx/index.php/becas-y-posgrados/becas-nacionales/formacion-para-la-administracion-publica.

Coubès, Marie-Laure, 2005, "Movilidad en la trayectoria laboral: transición entre sector formal-informal del empleo", en Coubès, Laure, Marie, Zavala de Cosío, María E. y Zenteno, René (eds.) Cambio demográfico y social en México del siglo XX, Cámara de Diputados, Tecnológico de Monterrey, El Colef, Porrúa, Tijuana B.C.

Coubès, Marie-Laure y Zenteno, René, 2005, “Transición hacia la vida adulta en el contexto mexicano: una discusión a partir del modelo normativo", en Coubès, Marie Laure, Zavala de Cosío, María E. y Zenteno, René (eds.) Cambio demográfico y social en México del siglo XX, Cámara de Diputados, Tecnológico de Monterrey, El Colef, Porrua, Tijuana B.C.

Du Bois-Reymond, Manuela y López-Blasco, Andreu, 2004, “Transiciones tipo yo-yo y trayectorias fallidas: hacia las políticas integradas de transición para los jóvenes europeos", en Revista de Estudios de Juventud, 65(4): 11-29.

El Colegio de la Frontera Norte, Instituto Nacional de Geografía e Informática y Universidad Autónoma de Baja California, 2013, Encuesta Demográfica Retrospectiva. (Consultado 17/abril/2015). Disponible en: https://www.colef.mx/ eder/?page_id=64.

Elder, Glen H., 1998, "The life course as developmental theory", en Child Development, 69(1): 1-12.

Elder, Glen; Johnson, Monica K. y Crosnoe, Robert, 2003, “The emergence and development of life course theory", en Mortimer, Jeylan T. y Shanahan, Michael J. (coords.) Handbook of the Life Course, Kluwer Academic / Plenum Publishers, Nueva York. 
Farkas, George, 1996, "Skills, habits, styles, and school success", en Human capital or cultural capital? Ethnicity and poverty groups in an urban school district, Aldine de Gruyter, New York.

Felmlee, Diane H., 1988, "Returning to School and Women's Occupational Attainment", en Sociology of Education, 61(1): 29-41.

García, Brígida y Pacheco, Edith, 2000, "Esposas, hijos e hijas en el mercado de trabajo de la Ciudad de México en 1995", en Estudios demográficos y urbanos, 15: 35-63.

Gaxiola, Hilda; Olmedo, Lourdes y Olivares, Sofía, 1997, "Un recorrido por la historia de la educación de adultos", en Veinte años de educación de adultos en la UAM Xochimilco. Memorias del primer encuentro, UAM-X, CSH, Depto. de Educación y Comunicación, México D.F.

Harley, Carolyn y Mortimer, Jeylan T., 2000, "Markers of transition to adulthood, socioeconomic status of origin, and trajectories of health", en Annals of New York Academy of Sciences, 896(1): 367-369.

Hausmann, Ricardo y Szekely, Miguel, 2003, "Inequality and the Family in Latin America", en Birdsall, Nancy, Kelley, Allen y Sinding, Steven (eds.), Population Matters. Demographic Change, Economic Growth, and Poverty in the Developing World, Oxford University Press, New York.

Haveman, Robert, y Wolfe, Barbara, 1993, “Children's prospects and children's policy”, en Journal of Economic Perspectives, 7: 153-174.

Hernández, Ana Karina y Vargas-Valle, Eunice, 2016, "Condiciones del trabajo estudiantil urbano y abandono escolar en México", en Estudios Demográficos y Urbanos, 31 (93): 663-696.

Hogan, Dennis P. y Astone, Nan Marie, 1986, "The transition to adulthood", en The Annual Review of Sociology, 12: 103-130.

Hogan, Dennis P., 1978, "The Variable Order of Events in the Life Course", en The American Sociological Review, 43(4): 573-586.

Hostetler, Andrew J., 2008, "Educational Careers, Returning to School and Work-Family Concerns", en Sloan Network Encyclopedia. 15 de mayo de 2014. Disponible en https://workfamily.sas.upenn.edu/wfrn-repo/object/uylg5zs719uz5e8g

Hostetler, Andrew J.; Sweet, Stephen y Moen, Phyllis, 2007, "Gendered career paths: A life-course perspective on returning to school", en Sex Roles, 56: 85-103.

Kirby, Peter G.; Biever, Joan L.; Martinez, Isaac G. y Gómez, John P., 2004, "Adults returning to school: The impact on family and work", en The Journal of Psychology, 138(1): 65-76.

Light, Audrey, 1995, "The effects of interrupted schooling on wages", en Journal of Human Resources, 30: 472-502.

Mier y Terán-Rocha, Marta y Rabell-Romero, Cecilia, 2001, “Condiciones de vida de los niños en México, 1960-1995” en Gómez de León Cruces, José y Rabell 
Romero, Cecilia (eds.) La población de México. Tendencias y perspectivas sociodemograficas hacia el siglo XXI, Consejo Nacional de Población, México.

Pacheco, Edith, 2005, "La movilidad ocupacional de los hijos frente a sus padres", en Coubès, Marie Laure, Zavala de Cosío, María E. y Zenteno, René (eds.) Cambio demográfico y social en México del siglo XX, Cámara de Diputados, Tecnológico de Monterrey, El Colef, Porrúa, Tijuana B.C.

Peinador Roldán, Rocío, 2005, "Migración interna y escolaridad durante la infancia y la adolescencia de tres generaciones en México”, en Mier y Terán, Martha y Rabell, Cecilia (eds.), Jóvenes y niños: un enfoque sociodemográfico, Miguel Angel Porrúa, México.

Pérez-Baleón, Guadalupe Fabiola y Lindstrom, David, 2014, "El regreso a la escuela: evidencias para México", en Estudios Demográficos y Urbanos, 29(3): 579619.

Rabe-Hesketh, Sophia y Skrondal, Anders, 2008, "Discrete time-survival", en Multilevel and Longitudinal Modeling Using Stata, Second Edition, College Station, Stata Press, Texas.

Rabell, Cecilia y Murillo, Sandra, 2016, "Coresidencia con los padres y bienestar en la infancia y la adolescencia”, en Coubès, Marie Laure, Solís, Patricio, y Zavala de Cosío, María E. (coords.) Generaciones, cursos de vida y desigualdad social en México, El Colegio de México, El Colegio de la Frontera Norte, México.

SEP, 2013, Sistema Educativo de los Estados Unidos Mexicanos, principales cifras, ciclo escolar 2011-2012, México, Secretaría de Educación Pública.

SEP, 2012, Reporte de la Encuesta Nacional de Deserción en la Educación Media Superior, México, Secretaría de Educación Pública (Consultado 20/abril/2014). Disponible en http:/www.decidetusestudios.sep.gob.mx/recursos/docs/ReporteEncuestaNacionalDesercionEMS.pdf

SEP, 2007, Estadistica Historica del Sistema Educativo Nacional, 08 de abril de 2008. Disponible en http://www.sep.gob.mx/work/appsite/nacional/index.htm.

SEP, 2009, El Gobierno Federal construye para que todos los servidores públicos terminen su educación básica, México, D.F., Secretaria de Educación Pública (Consultado 20/septiembre/2017). Disponible en www.sep.gob.mx/wb/sep1/ bol2170809\#.WcMQQsgjHIU.

SEP, 2004, "The development of education. National report of Mexico. Secretaría de Educación Pública", en Reporte presentado en la sesión 47 de la Conferencia Internacional sobre Educación (1-57). Geneva: IBE Geneva. (Consultado 20/marzo/2017). Disponible en http://www.ibe.unesco.org/National_Reports/ICE_2004/ mexico_ocr.pdf.

SEGOB, 2012, "DECRETO por el que se declara reformado el párrafo primero; el inciso c) de la fracción II y la fracción V del artículo 3”, 02/Septiembre/2012, Secretaría de Gobernación, en Diario Oficial de la Federación.

Settersten Jr., Richard A., 2003, "Age Structuring and the Rhythm of the Life Course", en Mortimer, Jeylan T. y Shanahan, Michael J. (coords.) Handbook of the Life Course, Kluwer Academic / Plenum Publishers, Nueva York. 
Vargas-Valle, Eunice D. y Potter, Joseph, 2011, "La transición demográfica y la asistencia escolar de los adolescentes en México", en Revista Mexicana de Estudios de la Juventud, 1: 11-30.

\section{RESUMEN CURRICULAR DE LOS AUTORES}

\section{Eunice D. Vargas Valle}

Doctora en Sociología por la Universidad de Texas en Austin y Maestra en Demografía por el Colegio de la Frontera Norte, donde trabaja como investigadora titular en el Departamento de Estudios de Población y editora de la revista Frontera Norte. También se ha desempeñado como Coordinadora Académica de la Maestría en Estudios de Población de esta institución y como Secretaria del Exterior de la Sociedad Mexicana de Demografía. Es miembro del Sistema Nacional de Investigadores Nivel 1. Sus líneas de investigación son: la demografía de la juventud, con énfasis en educación, migración y trabajo, y la dinámica poblacional en las fronteras de México. Tiene más de 20 publicaciones, capítulos y artículos en revistas académicas. Sus publicaciones más recientes se encuentran en The Social Science Journal (2018), Revista Mexicana de Sociología (2017) y Estudios Demográficos y Urbanos (2016).

Dirección electrónica: eunice@colef.mx

\section{Pedro Paulo Orraca Romano}

Doctor en Economía por la University of Sussex, Maestro en Economía por el Centro de Investigación y Docencia Económicas A.C. y Licenciado en Economía por la Universidad Autónoma de Baja California. Ha laborado en la Secretaría de Finanzas del Gobierno del Distrito Federal, en la London School of Economics y en la Universidad Autónoma de Baja California. Entre sus publicaciones destacan artículos en revistas como Oxford Development Studies, Latin American Economic Review, The Social Science Journal, Journal of Borderlands Studies, El Trimestre Económico y Estudios Económicos. Anteriormente obtuvo el Premio de Economía Joaquín Xirau Icaza, otorgado por El Colegio de México. Su línea de investigación se centra en las áreas de economía laboral, migración y economía transfronteriza. Es Investigador Titular adscrito al Departamento de Estudios Económicos de El Colegio de la Frontera Norte, Coordinador de la Maestría en Economía Aplicada en la misma institución y miembro del Sistema Nacional de Investigadores, Nivel 1.

Dirección electrónica: porraca@colef.mx

Artículo recibido el 2 de octubre de 2017 y aprobado el 21 de agosto de 2018. 